\title{
Numerical Analysis of Fracture Behavior of Surgical Cement in THP
}

\author{
Zouambi L ${ }^{1 *}$, Fekirini $\mathbf{H}^{2}$, Bourdim $\mathbf{M}^{1}$ and Serier $\mathbf{B}^{2}$ \\ ${ }^{1}$ LGIDD, Department of Mechanical Engineering, University Center of Relizane, 48000, Algeria \\ ${ }^{2}$ LMPM, Department of Mechanical Engineering, University of Sidi Bel Abbes, Sidi Bel Abbes 22000, Algeria
}

\begin{abstract}
In this study, we use the finite element method to analyse the behaviour of cracks emanating from microcavities in the bone cement, binding the cup to the bone, according to their size and position around the cavity, the position of the patient, the cavity's location and the inter-defects distance (cavity-crack, crack-crack). We show that the most unstable stress intensity factor, in mode I, when the crack located in the cement's centre and propagating along this thickness. This instability is all the more important that its size increases, tends towards the cavity, the cracks are located in a vicinity one to other and that the patient is in a squatting position. The predominant fracture mode, in mode I and II, depends on the crack's position priming site around the microcavities. This work allows the better understanding of the interconnection phenomena of the microcavities experimentally observed.
\end{abstract}

Keywords: Cement (PMMA); Crack; Position; Cavity; Stress intensity factor; Interaction

\section{Introduction}

In the hip, especially the stress mechanism is very complex: it is a combination of the compression, shear, torsion and tensile efforts. The intensity of these loads depends on the nature of the activities performed by the patient. These efforts, partially transmitted to the cement can be amplified by the defects existing in this material. Thus, one of the problems encountered by expert's cemented arthroplasty is the presence of defects in the cement. These defects (porosity, inclusions and cracks) are sources of additional stress by notch effect and are the main cause of loosening of total hip prostheses (THP) [1]. Three types of cracks [2] have been observed in experimental specimens in PMMA:

- Cracks initiated on voids which are practically always the cavities with blistering inside surface. They tend to connect several cavities;

- Cracks initiated from a pre-polymerized bubble;

- Cracks, from tearing, due to shrinkage and internal stresses during the polymerization of the cement, while it has not yet hardened.

It is known that the cracks that arise from porosity are the most dangerous defects in the cement and their propagation can lead to brutal fracture, and therefore, the loosening of the prosthesis. Benbarek et al. [3] numerically analysed, by element finites method, the behaviour of cracks emanating from microcavities in the cement, binding the cup to the bone. He showed that the SIF depends on the position of the cracks around the micro-defect. Bouiadjra et al. [4] showed that the microcracks positioned at $90^{\circ}$, relative to the horizontal axis of the cup, lead to the highest values of the SIF. Achour et al. [5] analysed by MEF, the behaviour of interfacial cracks between the cement and the implant in the proximal, medial and distal. They show that these cracks propagate preferentially in mode II. Serier et al. [6] demonstrated that the induced stress solicits the cement of the acetabulum in both tensile and compression, and the SIF depends on the crack orientation initiated in this binder. Murphy and Prendergast [7] showed that all cracks are emanating from porosities and their sense of direction depends on the intensity of the load applied (Figure 1).

The objective of this study is to better understand the phenomena of cracks emanating from cavities, interconnection of cavities by a crack emanating from a cavity and coalescence of cracks emanating from these defects observed experimentally [8]. These three cases were studied. To do this, the behaviour of cracks emanating from cavities of bone cement, binding together the cup to the bone, was analysed. This study was performed according to the orientation of the crack priming site in relation to axes of the cavity and their size, according to the position of the cavity and that of the patient, according to the interdistance between defects (cavity-crack and crack-crack). The fracture criterion used for this study is the stress intensity factor.

\section{Geometrical Model}

In this bi-dimensional FEM analysis of a right pelvic bone, the polyethylene cup having an inner diameter of $28 \mathrm{~mm}$ and external diameter of $54 \mathrm{~mm}$, it was fixed in a hemispherical acetabular cavity

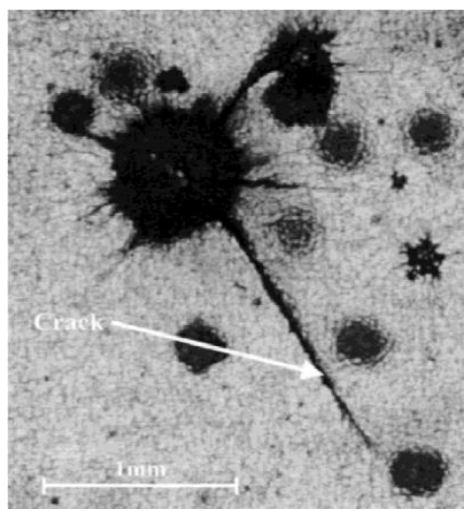

Figure 1: Cracks in acrylic bone cement observed under transmitted light.

*Corresponding author: Leila Zouambi, LGIDD, Department of Mechanical Engineering, University Center of Relizane, 48000, Algeria, Tel: +213 6685559 15; E-mail: zouambileila1071@gmail.com

Received August 02, 2017; Accepted August 18, 2017; Published August 28 2017

Citation: Zouambi L, Fekirini H, Bourdim M, Serier B (2017) Numerical Analysis of Fracture Behavior of Surgical Cement in THP. J Material Sci Eng 6: 371. doi: 10.4172/2169-0022.1000371

Copyright: (c) 2017 Zouambi L, et al. This is an open-access article distributed under the terms of the Creative Commons Attribution License, which permits unrestricted use, distribution, and reproduction in any medium, provided the original author and source are credited. 


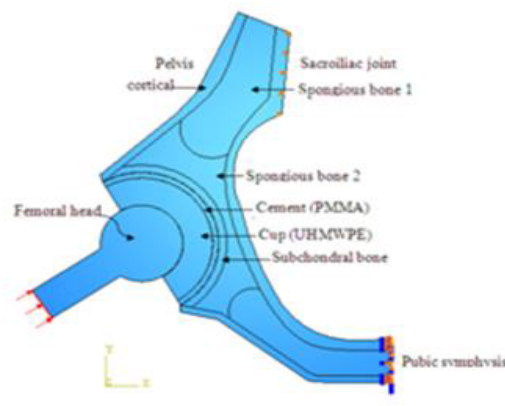

$0^{\circ}$

(a)
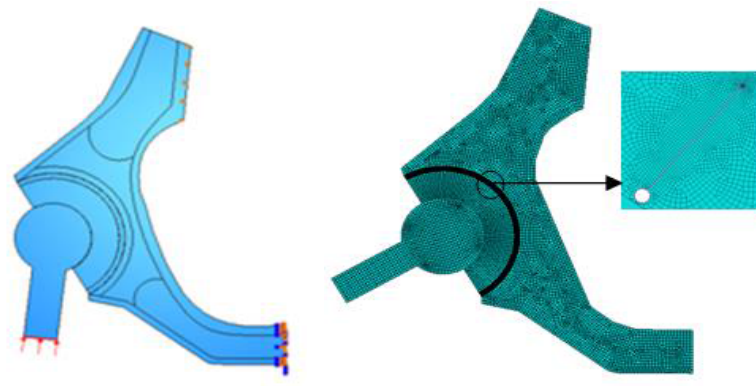

$50^{\circ}$

(b)

Figure 2: (a) Position of the femoral head relative to the cup axis $\left(0^{\circ}, 50^{\circ}\right)$ and limit conditions, (b) prosthesis mesh analyzed.

\begin{tabular}{|c|c|c|}
\hline & Young's Modulus (MPa) & Poisson's ratio $(v)$ \\
\hline Cortical bone & 17000 & 0.3 \\
\hline Spongious bone & 2 to 132 & 0.2 \\
\hline Subchondral bone & 2000 & 0.3 \\
\hline PMMA & 2300 & 0.3 \\
\hline UHMWPE & 690 & 0.3 \\
\hline Titanium alloy & 210000 & 0.3 \\
\hline \multicolumn{2}{|c|}{ Table 1: Materials properties [3]. } \\
\hline
\end{tabular}

through of cement PMMA with a thickness of $2 \mathrm{~mm}$. Materials properties were shown on Table 1.

The stress was applied to the femoral head is $30 \mathrm{MPa}[5]$ and was uniformly distributed. There has been a limited amount of research carried out into loading distribution acting on the acetabulum caused by the transfer of force from the femoral head. Three selected load cases were used with an average body weight of $70 \mathrm{~kg}$ assumed. The sacroiliac joint was fully fixed while the pubic joint was allowed to in sagittal plane, boundary conditions considered to be representative of anatomic configuration (Figure 2a) [8,9]. The contact between bone and cement and between cement and cup was taken as fully bounded, and between femoral head and cup was assumed to be frictionless under small sliding condition.

\section{Finite Elements}

The finite element method used in this work, for analyzing the mechanical behavior in fracture of bone cement. To do this we used the commercial software Abaqus [10].

The mesh refinement of the cement, cavity with $0.1 \mathrm{~mm}$ of diameter and a focused mesh was used near a crack tip is a great necessity for the structure analysis. Contributing to this field, we have analyzed the cracks behaviour in the cement. Our choice fell on quadrilateral elements to eight nodes (CPS8R) was established in the cement, triangular to three nodes (CPS3) for Ilium bone and quadrilateral to four nodes (CPS4R) for the other components of the prosthesis (Figure $2 b$ ).

\section{Results}

In this work, we analyse the behaviour of cracks emanating from cavities according to the position of their priming sites relative to the main axes of the cavities, the mechanical loading and the location of these voluminal defects in the bone cement. The presence of cavity in the bone cement causes its weakening [10]. The fracture criterion chosen for this analysis is the SIF (SIF). For this, we opted for a

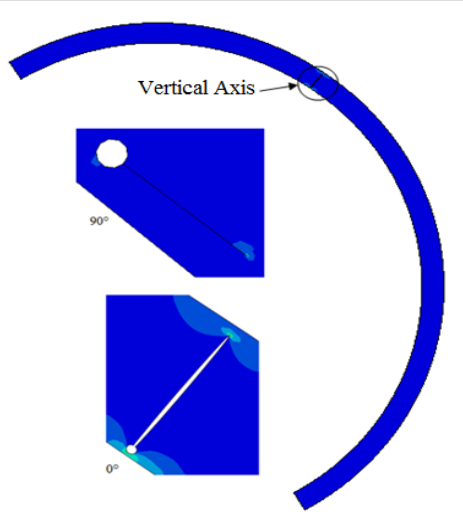

Figure 3: Cracking structure analysed.

squatting position of the patient defined by an orientation of $50^{\circ}$ to the axis of the neck of the femoral head relative to that of the cup.

\section{Effect of the crack's position}

Two cracks emanating from a cavity, initiated along its vertical axis and perpendicular to this axis, defined by their orientations relative to this reference mark of $0^{\circ}(1.75 \mathrm{~mm})$ and $90^{\circ}(5 \mathrm{~mm})$ respectively (Figure $3)$. These orientations characterize the positions of the priming site of the crack relative to the cavity. Recall that the behaviour of these two defects was analysed independently of each other. The results are represented in Figure 4. This last shows the evolution of the SIF according to the size of the two cracks in the opening mode. We note, however, that an increase in the first crack leads to an increase of the fracture criterion. In mode I, and from the negative values of this factor, the second is almost stable. Such behaviour clearly shows that the fracture mode of these defects differs totally from each other. Indeed, the first crack develops mainly in mode I, while the second mainly propagates in shear mode (mode II) as shown in Figure 5. This figure illustrates the variation of the SIF in mode II according to the size of the two cracks. These results indicate that the most unstable cracks are initiated along the thickness of the bone cement and this in spite of their small size. Such location of these defects can lead to loosening of the THP by fracture of the cement.

\section{Effect of the patient's position}

We are interested in the effect of the wearer's position of THP, characterized by the orientation of the implant axis relative to that of the cup, on the behaviour of cracks emanating from cavities (Figure 6). 


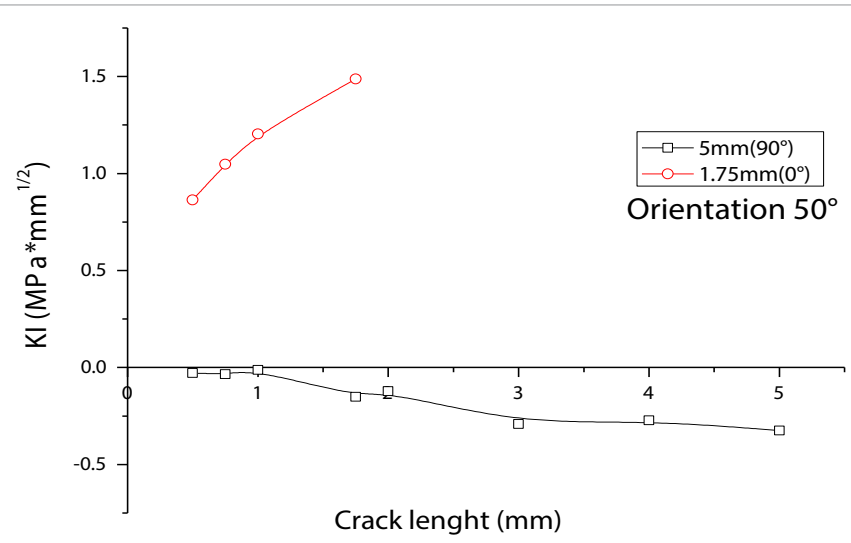

Figure 4: Variation of the SIF in mode I according to the crack size and its orientation relative to the vertical cavity axis.

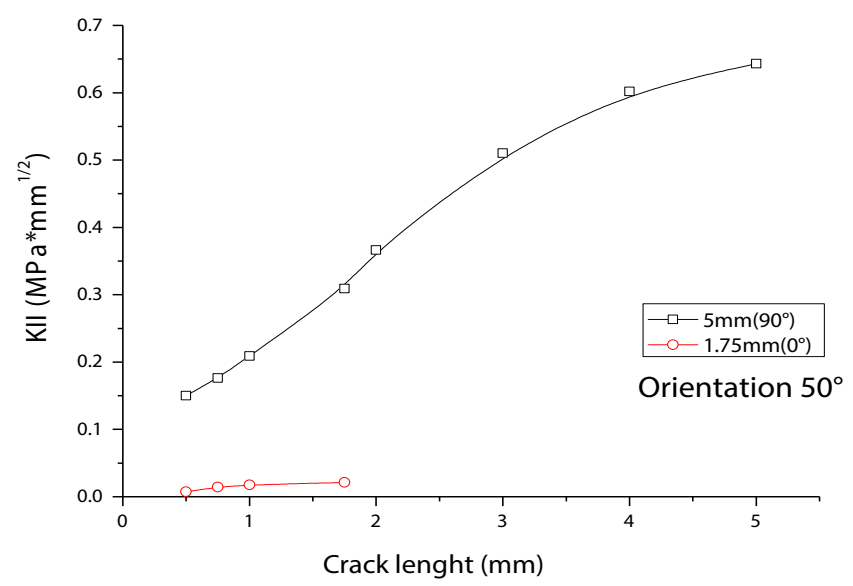

Figure 5: Variation of the SIF in mode II according to the crack size and its orientation relative to the vertical cavity axis.
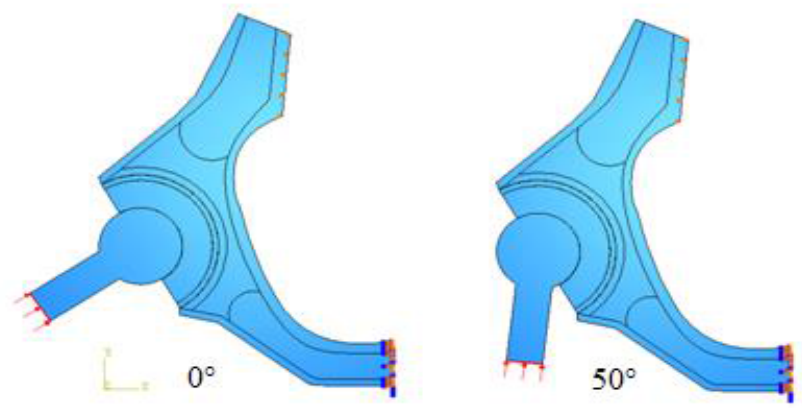

Figure 6: Positions studied of the human posture.

To do this, two positions were selected, standing and squatting, defined by angles of orientation $0^{\circ}$ and $50^{\circ}$, respectively. In Figure 7 a illustrates the variation of the SIF in the mode I, to the crack tip initiated along the thickness (vertical axis) of the cement according to these orientations. It shows that, from a standing position, results the factor most important and this, whatever the size of the crack emanating from the cavity. In mode II, the evolution of this fracture criterion based on the size of the crack and the nature of the patient's activity is shown in Figure $7 \mathrm{~b}$. The analysis of this figure clearly shows that this is from the squatting position that results the SIF the most important and this regardless of the length of the crack. Very low values of this factor show that such defect propagates preferentially in the opening mode.

The variation of the SIF, in mode I, resulting from a location of the crack perpendicular to the vertical axis of the cavity (crack oriented to $90^{\circ}$ ), according to the position of the implant axis relative to that of the cup is shown in Figure 8a. We note however, that independently of this position, growth, in mode I, of such a defect is stable. Such orientation of the crack favours its development in shear mode (mode II) as shown in Figure 8b. The results, shown in this figure, confirm those shown in Figure $7 \mathrm{~b}$. Indeed, comparatively to standing position, that squatting increases the risk of crack propagation by shearing of its lips (mode II). In other words, a squatting wearer's position of the THP increases the instability of such defects by increasing their kinetic of development.

The results obtained in this study show that the growth mode of cracks emanating from cavities of the bone cement depends on the location of their priming site and the nature of the wearer's activity of the THP.

\section{Effect of the position of cavities}

We have previously shown that the stresses induced in the cement are not distributed homogeneously along the bone cement and along its thickness. Such distribution, has led us to analyse the mechanical behaviour of cracks emanating from cavities located in three zones of the binder: two sides (left and right) are respectively noted LSP and RSP and a central noted CP (Figure 9). The results thus obtained are shown in Figure 10a. This last show the variation of the SIF, in mode I started at the crack tip, initiated from a cavity along its vertical axis according to its position (cavity) in the cement. We note that in mode I, the most unstable cracks are those from cavities on the central part of the bone cement. The SIF in mode I resulting from such cracks is the most important. The negative values of this factor illustrate that an initiated crack of a cavity located on the right side part is propagated essentially by shearing of its lips (mode II). The effect of the cavity's location where a crack is initiated and its size on the evolution of this fracture criterion mode II is shown in Figure 10b. This figure shows that in mode II, unstable cracks are initiated of cavities positioned on the side parts. Cracks emanating from cavities located in the centre and the left grow in modes I and II.

The variation of SIF in modes I and II according to the size of the crack initiated in $90^{\circ}$ relative to the vertical axis of the cavity and the position of the cavity in the cement is shown in Figure 11a and 11b. Such crack emanating from a cavity located in the centre and on the left of cement (CP and LSP) does not propagate in mode I (Figure 11a). Their growth is done essentially in mode II. We note however that the crack emanating from a cavity positioned on the right (RSP) does not grow in mode II. The values of the SIF, in mode I, in crack tip are higher than in mode II.

\section{Effect of interaction}

The results obtained previous show clearly that the level and distribution of the stresses generated in the bone cement, depend not only on the size of the cavities but also their voluminal fraction and the distance which separates them [10]. This led us to analyse the effects of interaction between a crack emanating from a cavity and another cavity, on the one hand, and between two initiated cracks of two cavities, on the other hand. These last are located along the cement and along its thickness characterized by orientations of $0^{\circ}$ and $90^{\circ}$ respectively. Critical conditions, that is to say, a location of the cavity on the central part of cement and a squatting position of the wearer of the THP, were 

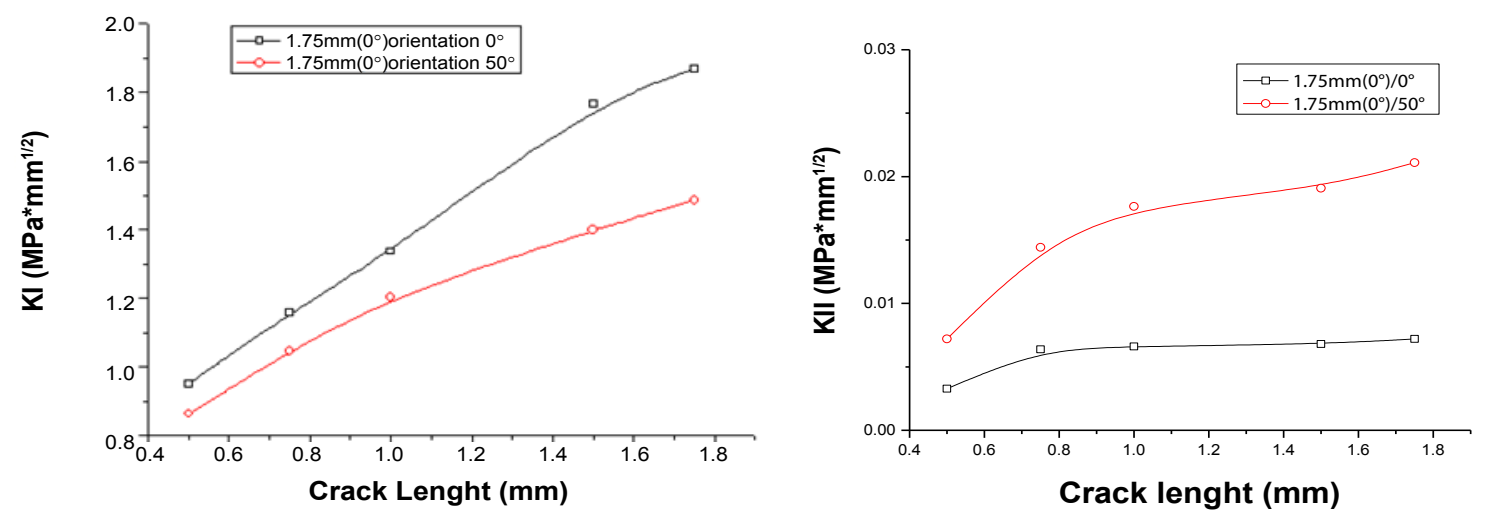

Figure 7: Variation of SIF to the crack tip oriented at $0^{\circ}$, according to the patient's position: (a) mode I and (b) mode II.
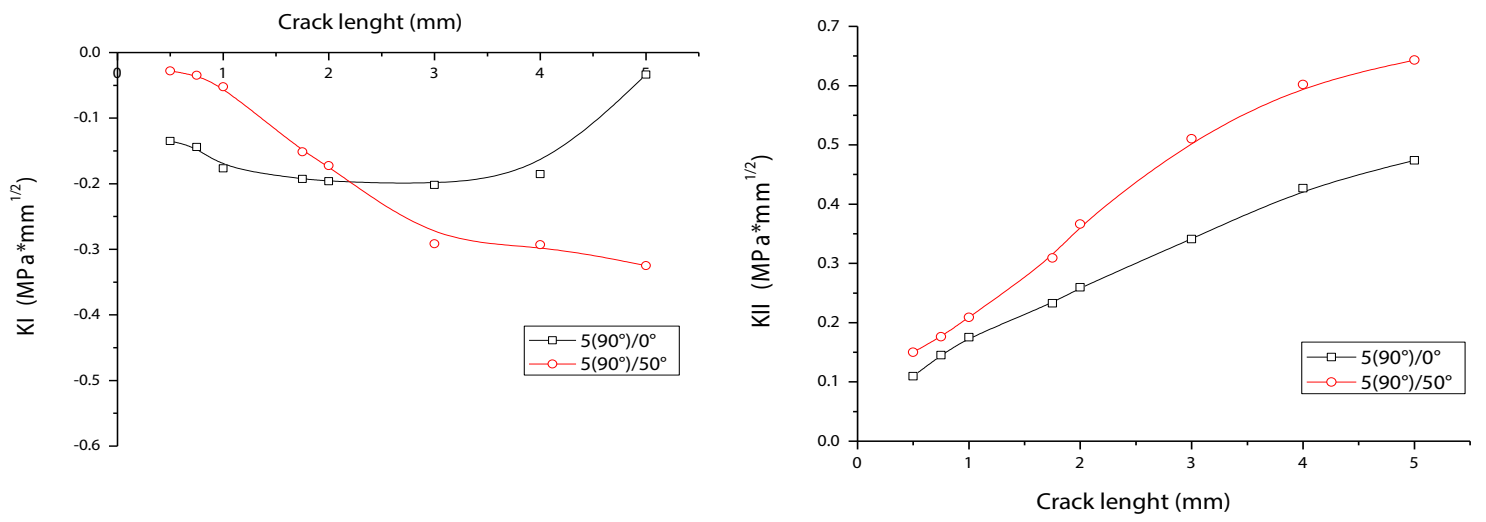

Figure 8: Variation of SIF to the crack tip oriented at $90^{\circ}$, according to the patient's position: (a) mode I and (b) mode II.

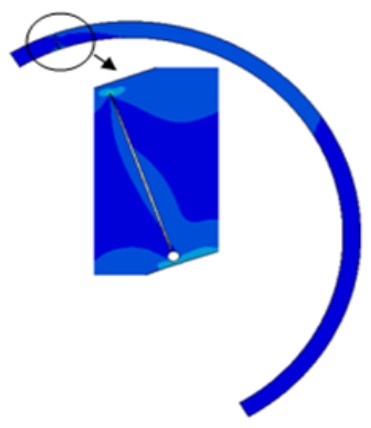

LSP

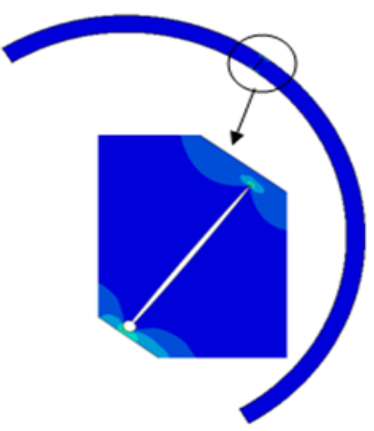

$\mathrm{CP}$

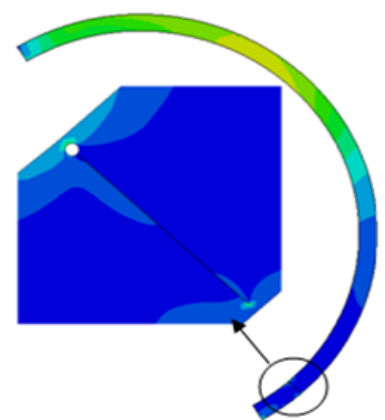

RSP

Figure 9: Analysed positions of the cavity in the cement.

selected for this study. The fracture criterion analysed is the SIF.

Crack-cavity: The effect of the distance separating a crack, emanating from a cavity 1 , and another cavity 2 (Figure 12), located in the bone cement, on the kinetics of propagation of this crack is analysed.

The results obtained are represented on Figure 13a. This figure shows the variation of the SIF in mode I according to the distance between an initiated crack from a cavity along the cement and its thickness (along the two principal axes of the cavity to $0^{\circ}$ and $90^{\circ}$ ) and another cavity. We note that this fracture criterion, resulting of a crack positioned at $0^{\circ}$, is all the more important that the crack emanating from the cavity, noted 1 , draws nearer to the second cavity noted 2 . A tendency of the crack towards the cavity leads to a very significant increase of this factor. This increase is characterized by the very rapid evolution of the slope of the curve $\mathrm{KI}=\mathrm{f}(\mathrm{a})$ of Figure 13a. comparatively with the crack positioned at $0^{\circ}$, developing essentially in mode I, the crack oriented at $90^{\circ}$ cannot propagates in mode I as the Figure $13 \mathrm{~b}$ shows it. According to this figure, we note that such a crack develops in pure mode II. A tendency of this crack towards the cavity, noted 2, 
Citation: Zouambi L, Fekirini H, Bourdim M, Serier B (2017) Numerical Analysis of Fracture Behavior of Surgical Cement in THP. J Material Sci Eng 6: 371. doi: 10.4172/2169-0022.1000371
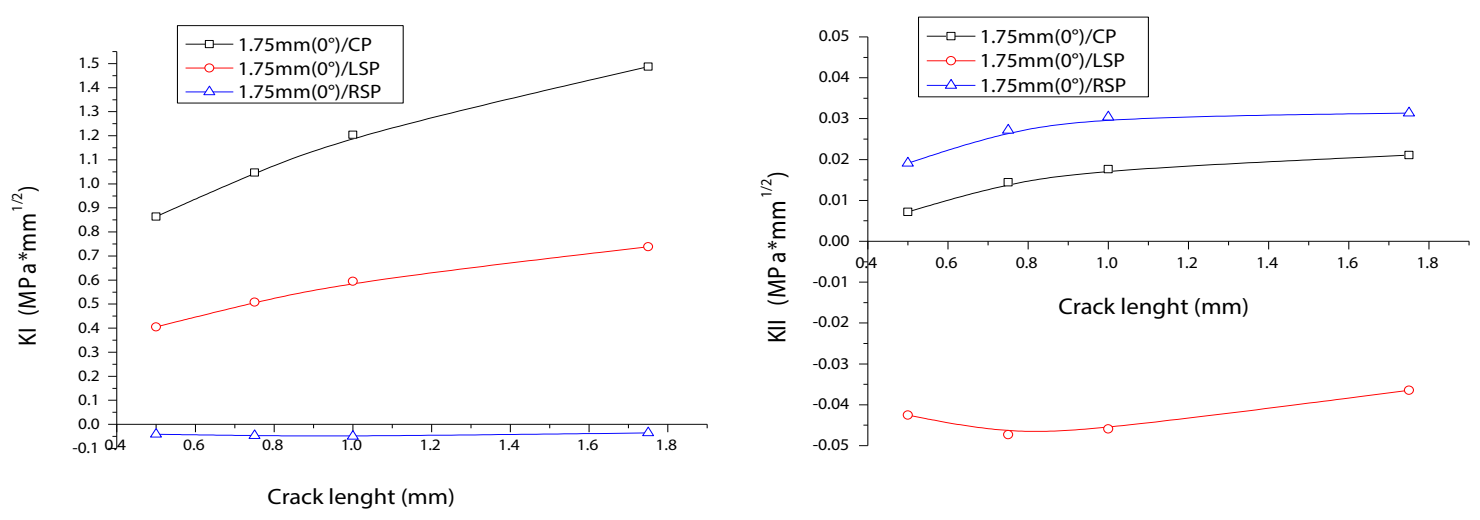

Figure 10: Variation of the SIF according to the position of the cavity: (a) mode I and (b) mode II.
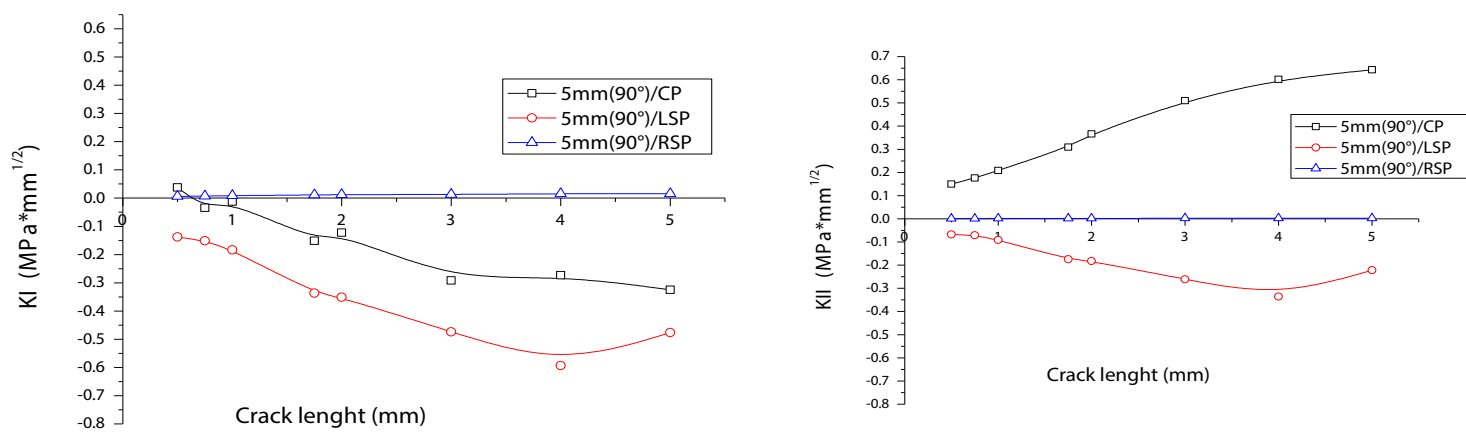

Figure 11: Variation of the SIF according to the position of the cavity and the orientation of the crack: (a) mode I and (b) mode II.

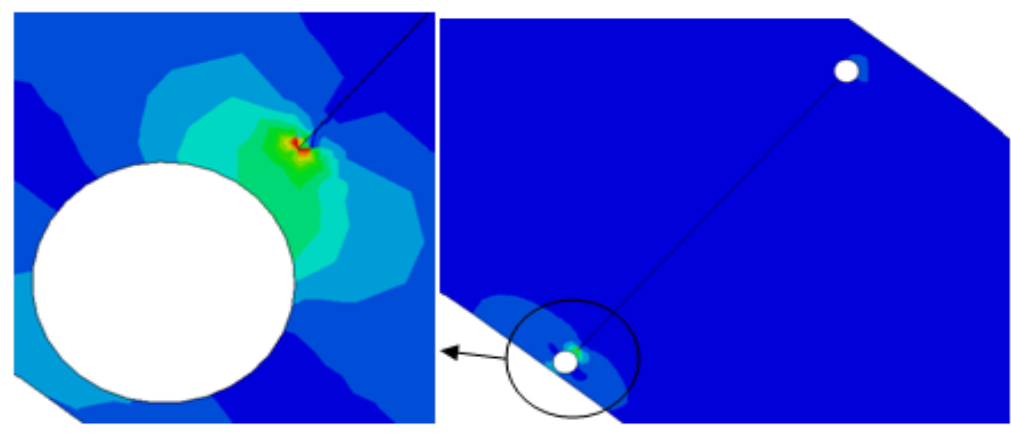

Figure 12: Interaction crack-cavity analysed.
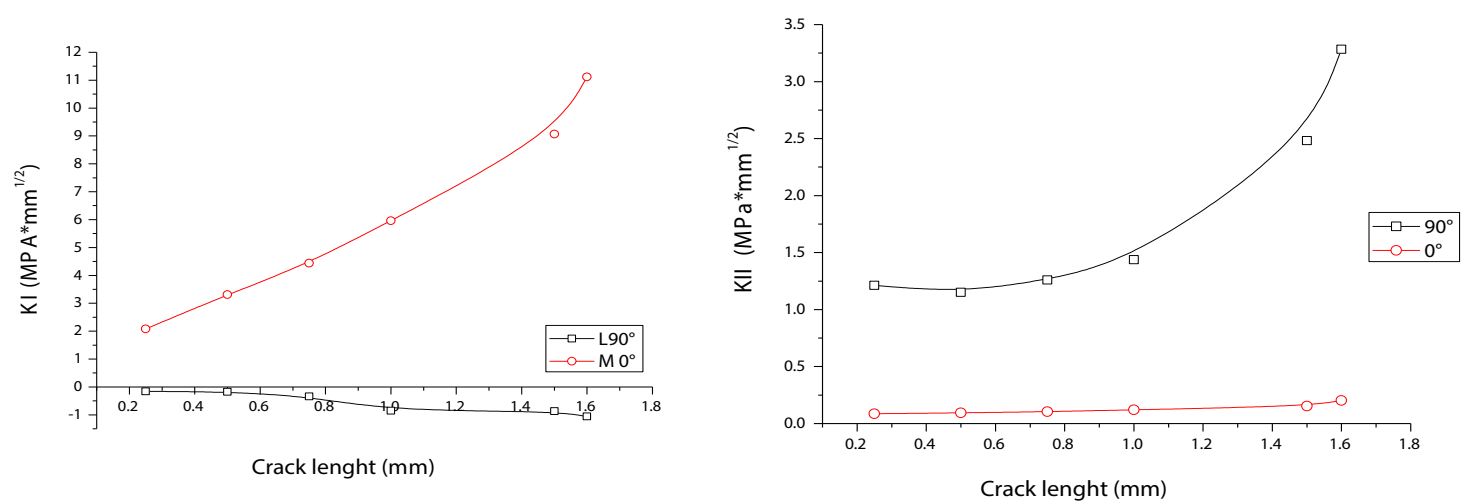

Figure 13: Crack-Cavity interaction: (a) mode I and (b) mode II. 
leads to a very rapid increase of this factor. Whatever the orientation of the crack and the fracture mode, the effect of crack-cavity interaction is paramount when defects are located in very close proximity to each other. In other words, the kinetics of propagation of these initiated cracks from the cavity, noted 1, grows strongly when there tend indefinitely toward the cavity, noted 2 . Such behaviour favours the interconnection of these two cavities observed experimentally [7].

The mechanical behaviour of cement is closely related to the voluminal fraction of cavities, characterized in our case by the distance between these voluminal defects. This interaction leads to an intensification of the stresses in the cement between these cavities whose level exceeds the threshold of fracture of tensile and compression of the binder. These stresses suffice therefore to initiate cracks emanating from cavities and propagate them brutally (very fast increase in the SIF in crack tip) leading thus to loosening of THP.

Crack-crack: In what follows, we analyse the interaction effect between a mobile crack emanating from a cavity 1 and another fixed emanating from another cavity 2 . These two defects are positioned along the cement and its thickness (Figure 14).

This is the SIF at the mobile defect tip which is analysed. In Figure $15 \mathrm{a}$, is shown the evolution of this fracture criterion in opening mode according to the size of the mobile crack. This factor grows slowly then rapidly with the advance of this crack. This behaviour is explained by changing the slope of the curve $\mathrm{KI}=\mathrm{f}$ (a) of Figure 15a. Comparatively to the results in Figure 7 for the same size of the crack, the SIF resulting from such a crack is much more important. In Figure 15b, is represented the variation of the SIF in mode II of the mobile crack according to the distance separating it from that fixed. The analysis of this figure shows that this crack is all the more unstable than when it tends to the fixed crack. This instability is characterized by a brutal increase of this fracture criterion. Comparatively to that illustrated on Figure 8 and for the same crack size, this factor is more important.

Our results clearly show that, whatever the fracture mode and the position of the cracks emanating from the cavities, the SIF is all the more important that the mobile crack tends toward that fixed. In other words, the interaction effect is determining when these two defects are located too close one to the other. This too fast increase of this factor leads to the interconnection of these two cracks and thus to their coalescence, phenomenon observed experimentally (Figure 1) [8]. This observation clearly shows the risk of interconnection is all the higher than the cavities are located very close one to another. That is in good agreement with the results obtained in this study.

\section{Conclusion}

The results obtained in this work show that:

- The fracture mode of cracks emanating from cavity depends on their priming sites. Cracks initiated along the bone cement propagate by shearing of its lips (Mode II) and those initiated along its thickness grow preferentially in opening mode (mode I);

- The nature of the wearer's activity of the THP determines the predominant fracture mode. The standing position favours the mode I and that squatting the mode II;

- The SIF at the crack tip emanating from cavity depends on its location in the cement. This fracture criterion resulting initiated cracks from cavities located on the left and right side parts of this binder is relatively low. The most unstable cracks emanating from a cavity located in the central zone of cement;

- The existence of a cavity in the direction of propagation of a
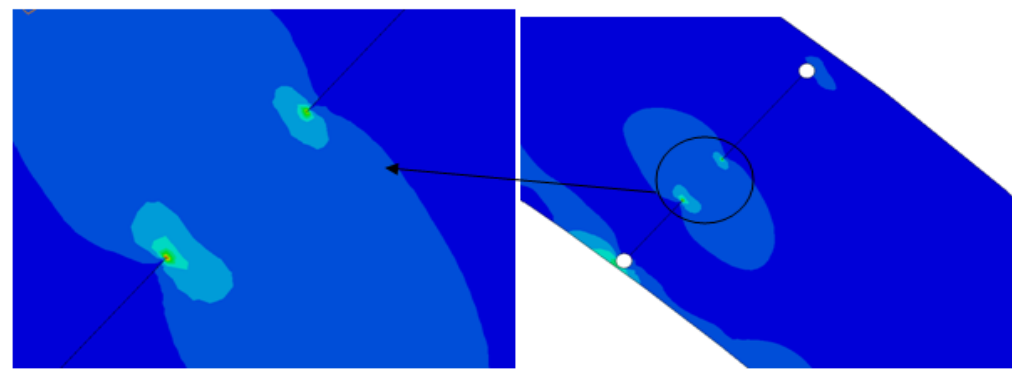

Figure 14: Mobile crack-Fixed crack Interaction studied.
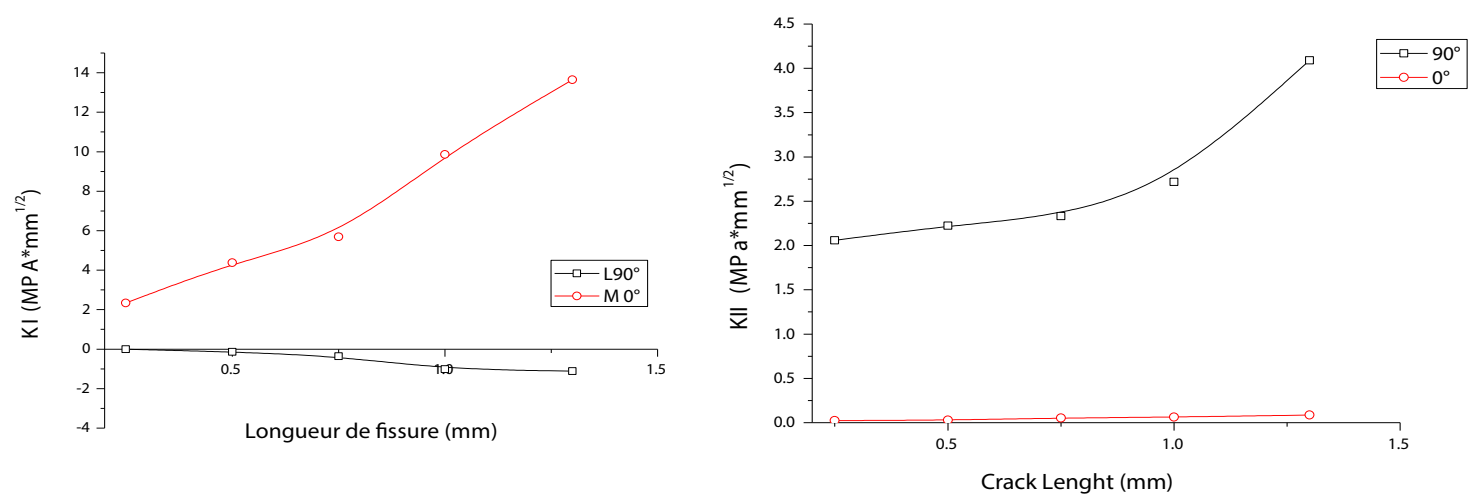

Figure 15: Mobile crack-fixed crack (0.25) Interaction, mode I. 
Citation: Zouambi L, Fekirini H, Bourdim M, Serier B (2017) Numerical Analysis of Fracture Behavior of Surgical Cement in THP. J Material Sci Eng 6: 371. doi: 10.4172/2169-0022.1000371

Page 7 of 7

crack emanating from another cavity accelerates its instability. this one becomes more marked when the crack draws nearer indefinitely the cavity;

- The growth of a crack emanating from a cavity toward another fixed initiated from another cavity favours very strongly its instability. The kinetics of growth increases very rapidly when the crack tends towards the fixed crack.

\section{References}

1. ABAQUS (2004) Ver 6-5, User guide, Cornell University.

2. Achour T, Tabeti MSH, Bouziane MM, Benbarek S, Bouiadjra BB, et al. (2010) Finite element analysis of interfacial crack behaviour in cemented total hip arthroplasty. Computational Materials Science 47: 672-677.

3. Benbarek S, Achour T, Serier B, Bouiadjra BB, Belarbi A (2007) FE analysis of the behaviour of microcracks in the cement mantle of reconstructed acetabulum in the total hip prosthesis. Computational Materials Science 40: 485-491.

4. Bouiadjra B, Achour T, Belhouari M, Serier B, Benbarek S (2007) Finite element analysis of the behaviour of crack emanating from microvoid in cement of reconstructed acetabulum. Materials Science and Engineering: A 457: 385-391.

5. Achour T, Serier B, Bouiadjra BB, Benbarek S (2009) Analysis of the effect of the forces during gait on the fracture behaviour in cement of reconstructed acetabulum. Computational Materials Science 46: 267-274

6. Serier B, Fekirini H, Bouiadjra BB, Zouambi L (2013) Effect of the CavityCavity Interaction on the Stress Amplitude in Orthopedic Cement. Journal of Biomaterials and Nanobiotechnology 4: 30 .

7. Murphy BP, Prendergast PJ (2002) The relationship between stress, porosity and nonlinear damage accumulation in acrylic bone cement. Journal of Biomedical Materials Research Part A 59: 646-654.

8. Hooy-Corstjens CS, Govaert LE, Spoelstra AB, Bulstra SK, Wetzels GM, et al. (2004) Mechanical behaviour of a new acrylic radiopaque iodine-containing bone cement. Biomaterials 25: 2657-2667.

9. Pernod P, Hernigou P (1996) Aspect morphologique de la porosité du ciment Actualités en Biomatériaux. Romillat, Paris 3: 235-240.

10. Yaszemski MJ, Biomaterials in Orthopedics, Mayo Clinic, Rochester, Minnesota, U.S.A. 